\title{
Synthesis of nanocrystalline CdS thin films in PVA matrix
}

\author{
R DEVI*, P PURKAYASTHA ${ }^{\dagger}$, P K KALITA ${ }^{\dagger}$ and B K SARMA \\ Department of Physics, Gauhati University, Guwahati 781 014, India \\ ${ }^{\dagger}$ Department of Physics, Guwahati College, Guwahati 781 021, India
}

MS received 14 August 2006; revised 25 January 2007

\begin{abstract}
Nanocrystalline thin films of CdS are deposited on glass substrates by chemical bath deposition technique using polyvinyl alcohol (PVA) matrix solution. Crystallite sizes of the nanocrystalline films are determined from broadening of X-ray diffraction lines and are found to vary from 5.4-10.2 $\mathrm{nm}$. The band gap of the nanocrystalline material is determined from the UV spectrograph. The absorption edge is shifted towards the lower wave length side (i.e. blue shift) and are found to be within the range from $2 \cdot 48-2.8 \mathrm{eV}$ as grain sizes decrease from 10.2-5.4 $\mathrm{nm}$. This is also supported by the spectral response curves. An increase of molarity decreases the grain size which in turn increases the band gap.
\end{abstract}

Keywords. Chemical method; UV spectra; spectral response; energy band gap.

\section{Introduction}

Thin films now occupy a prominent place in basic research and solid state technology. The use of thin film semiconductors has attracted much interest in an expanding variety of applications in various electronic and optoelectronic devices due to their low production costs. Cadmium sulphide $(\mathrm{CdS})$ is one of the most promising II-VI compound materials because of its wide range of application in various optoelectronic (Iyechika et al 1988; Dhumure and Lokhande 1993; Lee et al 2003), piezo-electronic (Kerk and Kelly 1964; Stefko 1991) and semiconducting devices. The thin films of $\mathrm{CdS}$ are of considerable interest for their efficient use in the fabrication of solar cells (Chu et al 1992; Britt and Ferekids 1993). In recent years nanocrystalline thin films of different II-VI compound semiconductors have been widely synthesized and studied. Nanocrystalline thin films are also polycrystalline in nature but with sizes of crystallites of the order of a few nanometers. Many properties of nanocrystalline materials are found to deviate from those of coarse grained polycrystalline materials with the same average chemical composition. These deviations result from dimensionality of nanometer sized grains and numerous interfaces between adjacent crystallites (Tong and Zhu 2006). A major research goal of recent years is to understand the size dependent properties of nanocrystalline materials and as a result considerable amount of work on synthesis and characterization of nanocrystalline thin films are being carried out (Nandakumar et al 1997; Breen et al 1998; Behera et al 2000; He et al 2003).

\footnotetext{
*Author for correspondence (kuhkhal@yahoo.com)
}

Out of several methods for deposition of nanocrystalline thin films, chemical bath deposition technique in polymer matrix is relatively simple, cost effective and suitable for deposition of film on large area substrate. In the present communication, results of preparation and characterization of nanocrystalline thin films of $\mathrm{CdS}$ within the self organized pores of PVA via a CBD process are reported.

\section{Experimental}

Nanocrystalline thin films were deposited on glass substrates by CBD technique. The deposition was carried out in a mixture of matrix solution and thiourea. The matrix solution was prepared by adding cadmium sulphate to an aqueous solution (2\%) of PVA with constant stirring at a constant temperature $\left(70^{\circ} \mathrm{C}\right)$ maintained for $90 \mathrm{~min}$. Five different concentrations of cadmium sulphate $(0.1 \mathrm{M}, 0.25 \mathrm{M}$, $0.5 \mathrm{M}, 0.75 \mathrm{M}$ and $1 \mathrm{M}$ ) were used to get five different matrix solutions. The solution was then left for $24 \mathrm{~h}$ to get a transparent liquid indicating complete dissolution of cadmium sulphate. The $\mathrm{pH}$ of the solution was maintained at around 11 by slowly adding $\mathrm{NH}_{4} \mathrm{OH}$ solution. Then the equimolar solution of thiourea was added to the mixture solution and glass substrates were introduced vertically into the solution with the help of a substrate holder. Within a few minutes colour of the solution changed to yellow. The substrates were kept in the solution for 18-24 h at room temperature for deposition of films. After deposition, the substrates were taken out and thoroughly washed and rinsed with doubly distilled water and dried in air. Grain sizes of the films were then measured by X-ray diffractometer (Philips X-pert Pro diffractometer (PW 1830)) at room temperature with $\mathrm{CuK} \alpha$ radiation. Surface morpho- 
logical study of the CdS thin films were done using scanning electron microscope (SEM) operating with an accelerating voltage of $20 \mathrm{kV}$. Grain sizes of the films were determined from SEM photographs at different magnifications. IR spectra were recorded using Perkin-Elmer instrument series II (2400-CHNS/0). Samples were prepared by mixing the dried $\mathrm{CdS}$ nanoparticles with $\mathrm{KBr}$ and pressing them into pellets. Optical absorption spectra at $300 \mathrm{~K}$ were obtained using a UV visible spectrophotometer (VARIAN CARY 300 scan) in the wavelength range $300-700 \mathrm{~nm}$. For spectral response studies, the current measurement was done with the help of a high impedance $\left(\sim 10^{14} \Omega\right)$ ECIL (electrometer amplifier (EA815)). A series of highly stable dry cells each of emf $9 \mathrm{~V}$ was used for providing the applied bias. A tungsten halogen lamp with a parabolic focusing mirror was used as the light source. A set of metal interference filters was used to get the monochromatic radiations of different wavelengths. The intensity of illumination is measured with a sensitive APLAB luxmeter.

\section{Results and discussion}

All the measurements were done at room temperature for the films with different molarity deposited by chemical route. The thickness of the films were 1500-1800 $\AA$, measured by the multiple beam interferometry method.

\subsection{XRD studies}

The X-ray diffractogram of CdS films show broadened diffraction profiles (figure 1). It is observed that XRD patterns show a preferred orientation along (002) plane. The crystallite size of the nanocrystalline films is estimated using the Scherrer formula

$$
D=K \lambda / \beta_{2 \theta} \cos \theta,
$$

where $K$ is a constant taken to be $0 \cdot 94, \lambda$ the wavelength of X-ray used $(\lambda=1.54 \AA)$ and $\beta_{2 \theta}$ the full width at half maximum of (002) peak of XRD pattern, Bragg angle, $2 \theta$, is around $26 \cdot 5^{\circ}$. The values of crystallite sizes of the films are shown in table 1 . The crystallite sizes were found to be within the range $5 \cdot 4-10 \cdot 2 \mathrm{~nm}$. It is observed that crystallite size decreases with increase in molarity.

\subsection{SEM analysis}

Scanning electron microscopy is a convenient technique to study the microstructure of thin films. Figure 2 shows the surface morphology of $\mathrm{CdS}$ thin films deposited at room temperature for five different molarities observed by SEM. From the micrographs, it is observed that the 'as-deposited' films are not uniform throughout all the regions. But the films are without any void, pinhole or cracks and that they cover the substrates well. From the figure, we clearly observe the small nanosized grains engaged in a fibrouslike structure, which clearly indicates the nanocrystalline nature along with some amorphous phase of $\mathrm{CdS}$ thin films. From these images, it can be seen that the grain sizes of the films are not uniform. Therefore, average grain sizes were estimated from different grains within the film and found to be about $44 \cdot 4-103.2 \mathrm{~nm}$. The values of grain sizes measured from SEM pictures are presented in table 1. It is quite clear from the results that the average grain
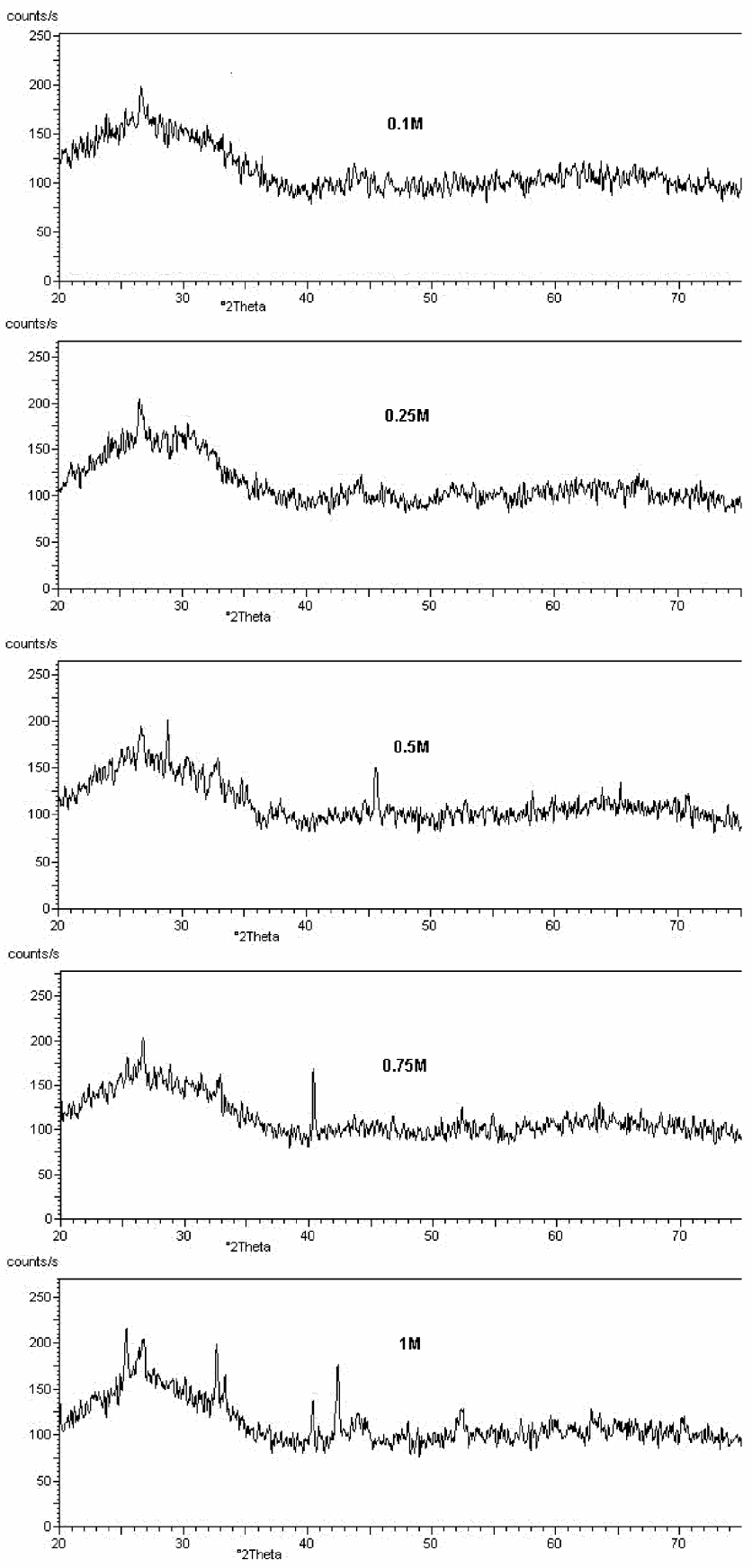

Figure 1. XRD patterns of CdS nanocrystalline films for different molarities. 
Table 1. The values of crystallite sizes and band gaps for CdS nanocrystalline films having different molarities.

\begin{tabular}{lccccc}
\hline Film no. & Molarity & $\begin{array}{c}\text { Grain size }(\mathrm{nm}) \\
\text { from XRD }\end{array}$ & $\begin{array}{c}\text { Grain size }(\mathrm{nm}) \\
\text { from SEM }\end{array}$ & $\begin{array}{c}\text { Band gap (eV) } \\
\text { from UV }\end{array}$ & $\begin{array}{c}\text { Band gap (eV) } \\
\text { from spectral response }\end{array}$ \\
\hline 1 & $0 \cdot 1 \mathrm{M}$ & $10 \cdot 2$ & 103.2 & 2.48 & 2.53 \\
2 & $0 \cdot 25 \mathrm{M}$ & $8 \cdot 2$ & 68 & 2.52 & 2.61 \\
3 & $0 \cdot 5 \mathrm{M}$ & $7 \cdot 0$ & 61.9 & 2.6 & 2.66 \\
4 & $0.75 \mathrm{M}$ & $5 \cdot 8$ & 53.3 & 2.7 & 2.75 \\
5 & $1 \mathrm{M}$ & 5.4 & 44.4 & 2.8 & 2.89 \\
\hline
\end{tabular}
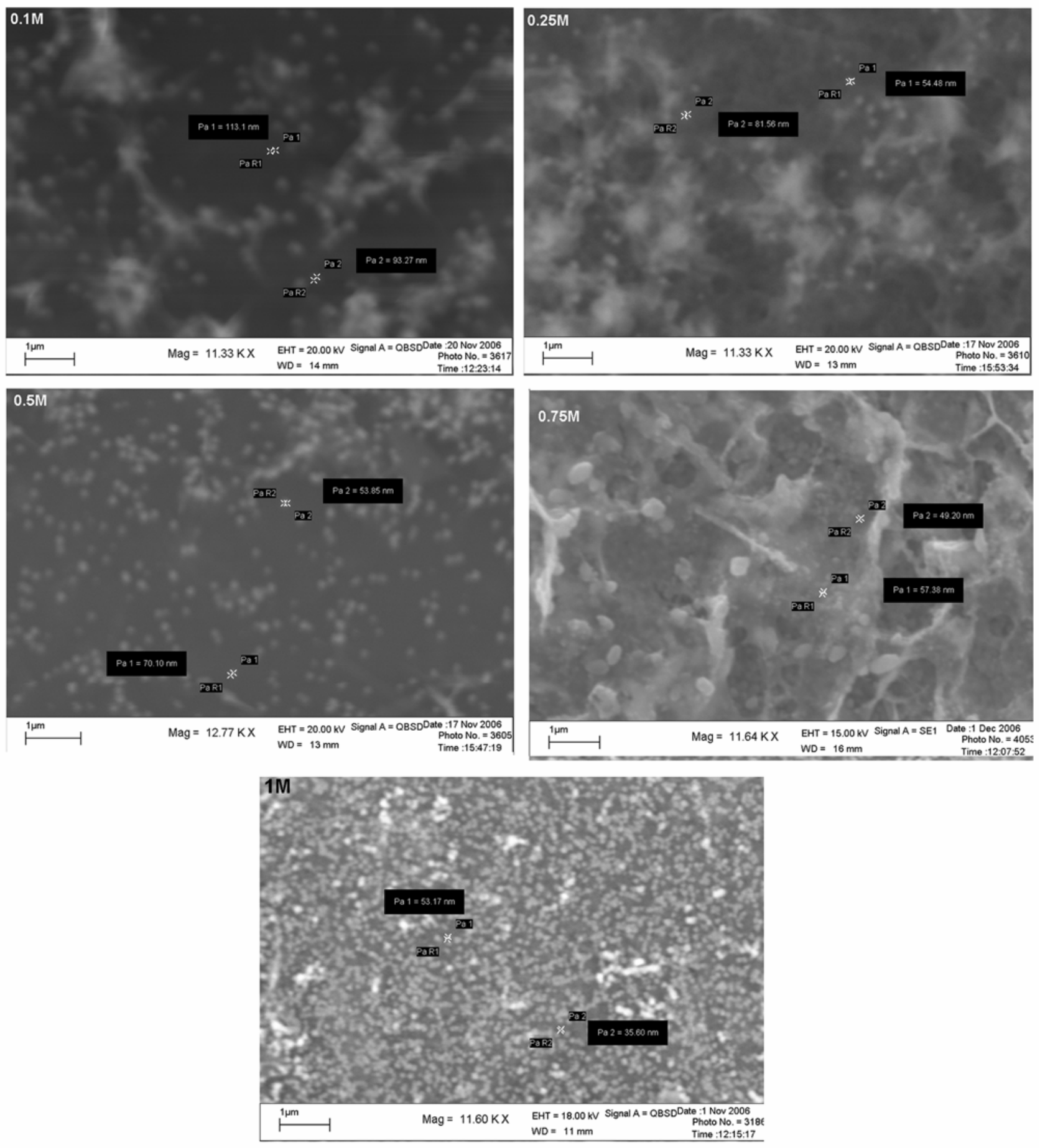

Figure 2. SEM photographs of CdS nanocrystalline films for different molarities at almost equal magnifications. 


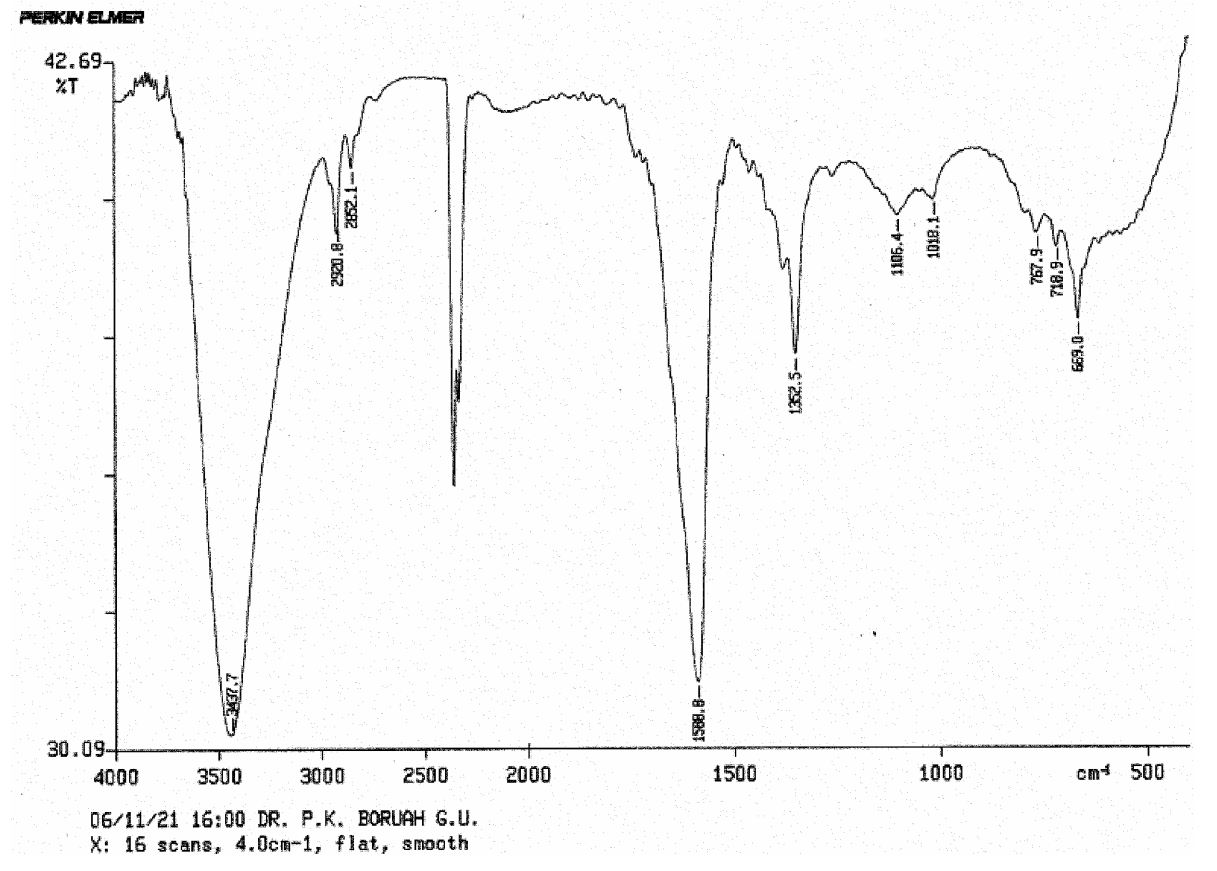

Figure 3. IR spectra of CdS nanoparticles for $0.5 \mathrm{M}$ molarity.

Table 2. Vibrational assignments of CdS nanomaterials with positions and intensities of absorption shown by IR spectra for a typical film $(0.5 \mathrm{M})$.

\begin{tabular}{lll}
\hline Positions $\left(\mathrm{cm}^{-1}\right)$ & Intensities & \multicolumn{1}{c}{ Assignments } \\
\hline $3437 \cdot 7$ & Strong & $\mathrm{OH}$ stretching, $v_{1} v_{3} \mathrm{H}_{2} \mathrm{O}$ \\
$2920 \cdot 8$ & Doublet & $\mathrm{C}-\mathrm{H}$ stretching \\
$2852 \cdot 1$ & & \\
$1588 \cdot 8$ & Strong & $\mathrm{C}-\mathrm{C}$ stretching \\
$1352 \cdot 5$ & Medium & $\mathrm{C}-\mathrm{O}$ stretching, $v_{2} \mathrm{H}_{2} \mathrm{O}$ \\
$1106 \cdot 4$ & Weak & $\mathrm{SO}_{4}^{-}$as trace \\
$1018 \cdot 1$ & & \\
$669 \cdot 0$ & Medium & $\mathrm{Cd}-\mathrm{S}$ stretching \\
$718 \cdot 9$ & & \\
\hline
\end{tabular}

size estimated by SEM is quite larger than the average grain size measured by XRD.

\subsection{IR spectra}

IR spectra of CdS nanoparticles for different molarities were similar, hence one graph of a typical deposition $(0 \cdot 5 \mathrm{M})$ is presented in figure 3 . The IR frequencies along with the vibrational assignments for $\mathrm{CdS}$ nanoparticles are given in table 2. The band at $3437.7 \mathrm{~cm}^{-1}$ are due to $\mathrm{OH}$ stretching vibrations of water molecules. The bending vibrations of water molecules appeared at $1588.8 \mathrm{~cm}^{-1}$, $\mathrm{C}-\mathrm{C}$ stretching, and a COO-unsymmetrical stretching at $1352.5 \mathrm{~cm}^{-1}$ appeared, which were induced by the trace

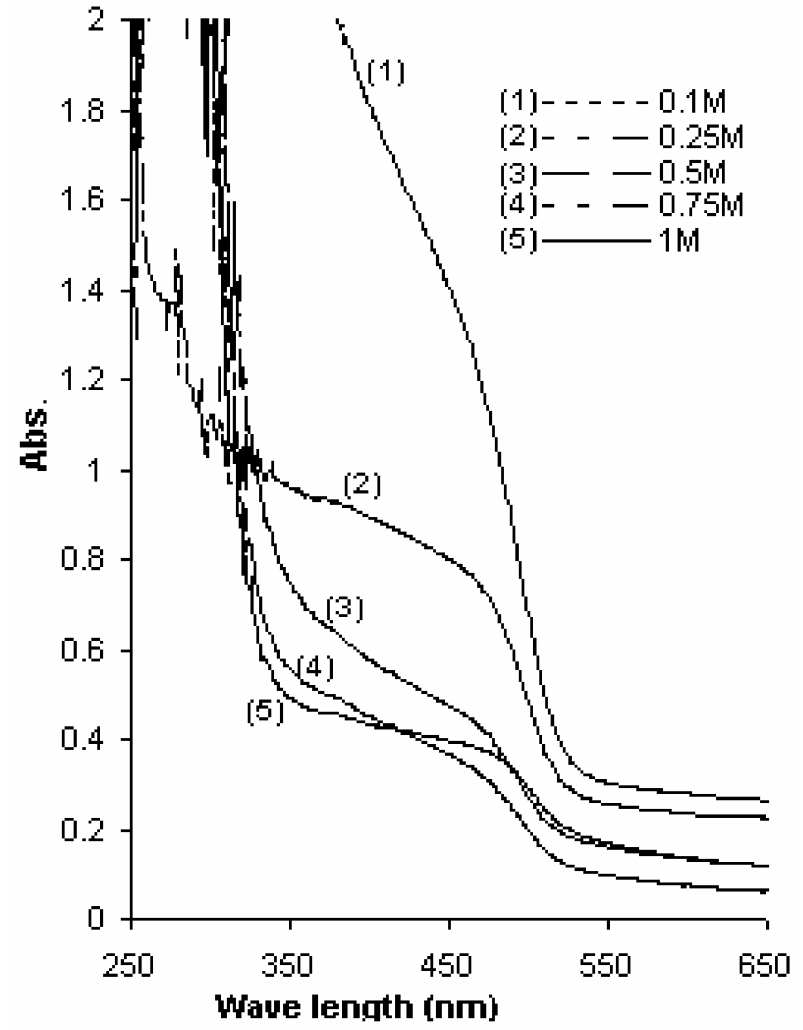

Figure 4. UV-absorption spectra of CdS nanocrystalline films for different molarities.

amount of PVA present as impurities bound to the $\mathrm{CdS}$ core. CdS particles showed two stretching bands, asymmetric and symmetric, at $2920 \cdot 8 \mathrm{~cm}^{-1}$ and $2852 \cdot 1 \mathrm{~cm}^{-1}$, associating with $\mathrm{C}-\mathrm{H}$ stretching (Tang et al 2005). Trace 
amount of $\mathrm{SO}_{4}$ as impurity is seen as there are small absorptions around $1105 \mathrm{~cm}^{-1}$. Absorptions at $1106.4 \mathrm{~cm}^{-1}$ and $1018 \cdot 1 \mathrm{~cm}^{-1}$ were assigned to $\mathrm{SO}_{4}^{-}$(Williams and Fleming 2004). At $669.0 \mathrm{~cm}^{-1}$ and $718.9 \mathrm{~cm}^{-1}$, there are medium to strong bands which have been assigned to $\mathrm{Cd}-\mathrm{S}$ stretching (Periasamy et al 1997). The vibration absorption peak of the $\mathrm{Cd}-\mathrm{S}$ band, which should be at $405 \mathrm{~cm}^{-1}$ and $265 \mathrm{~cm}^{-1}$, as reported by other workers (Martin et al 1982; He et al 2003) could not be observed since it was beyond the extent of our measurement.

\subsection{Absorption studies}

The UV absorption spectra of CdS/PVA thin films for different molarities taken at room temperature are shown in figure 4. From the spectrograph the absorption edge of the samples are found to occur in the range 350-450 nm for nanocrystalline films. Absorption spectrum shows a clear shift to the lower wavelength side over bulk crystallites at $\sim 515 \mathrm{~nm}$. This blue shift of the absorption edge indicates decrease of the crystallite sizes of the samples. CdS is a typical direct band gap semiconductor. According to Tauc relation, the absorption coefficient for direct band material is given by (Tauc 1974; Sharma et al 1992)

$$
\alpha=c\left(h v-E_{\mathrm{g}}\right)^{1 / 2} / h v,
$$

where $\alpha$ is the absorption coefficient, $c$ a constant, $h v$ the photon energy and $E_{\mathrm{g}}$ the band gap. The spectrographs were studied using the standard relation (2). A graph between $h v$ vs $(\alpha h v)^{2}$ is plotted and shown in figure 5. The extrapolation of straight line to $(\alpha h v)^{2}=0$ axis gives the value of the energy band gap of film materials. The band gap of the films are determined from the plots which are found to be within $2 \cdot 5-2 \cdot 8 \mathrm{eV}$. The band gap increases with the decrease of crystallite size. This observation of increment in band gap is approximately inversely propor-

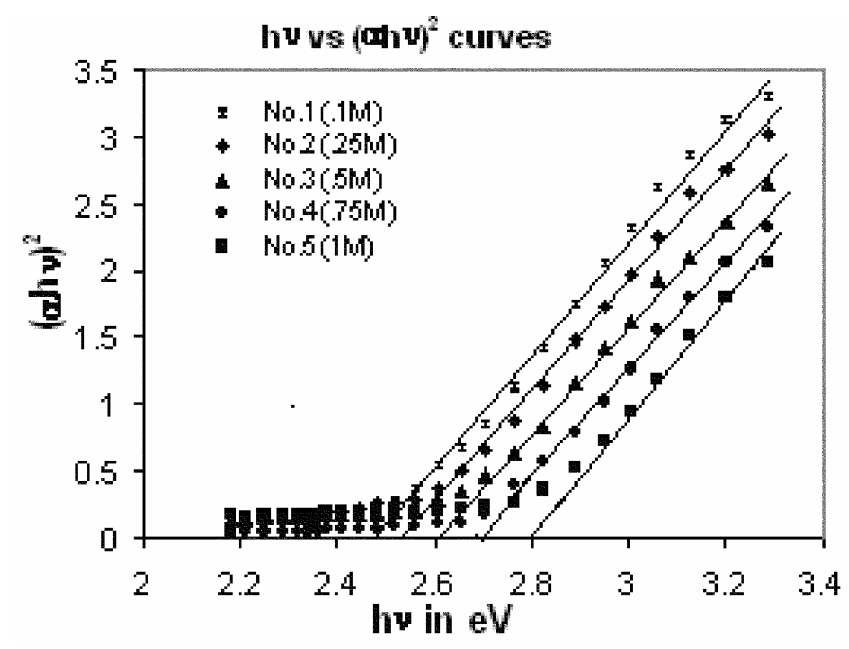

Figure 5. Energy band gap determination of $\mathrm{CdS}$ films for different molarities from the $h v$ vs $(\alpha h v)^{2}$ graph. tional to the square of the crystallite size based on the effective mass approximation (Brus 1986).

\subsection{Study of spectral response (optical band edge)}

By investigating the spectral response properties of thin film semiconductor for light of energy greater than the forbidden energy gap, it is possible to obtain information on its band structure (Kisiel et al 1976). Photoconductive materials generally exhibit spectral distribution curve having more or less sharp peaks in the vicinity of the absorption edge. The variation of photocurrent due to monochromatic radiation is observed at different applied biases in the chemically deposited CdS thin films and a typical curve at applied bias, $27 \mathrm{~V}$, for different molarity is shown in figure 6 . Here the photocurrent density is defined as

$$
J_{\text {ph }}=J_{\mathrm{L}}-J_{\mathrm{D}}
$$

where $J_{\mathrm{L}}$ is the current density due to monochromatic radiation and $J_{\mathrm{D}}$ the corresponding dark current density. During the measurements, a constant photon flux density for each selected wavelength is used by keeping the intensity fixed at $100 \mathrm{~lx}$ (normalized intensity). From the plots of photocurrent vs wavelength of monochromatic radiation $(\lambda=350-600 \mathrm{~nm})$ shown in figure 6 , it is observed that there is a maximum absorption peak at $425-491 \mathrm{~nm}$. The band gaps were determined from the absorption peak. From this absorption edge the corresponding band gap is found to be $2.89-2.53 \mathrm{eV}$. For different molarities the amount of absorption is different. The band gap increases with the increase in molarity. It is to be noted that the difference of band gap determined by UV absorption spectra and spectral response studies reduces significantly along

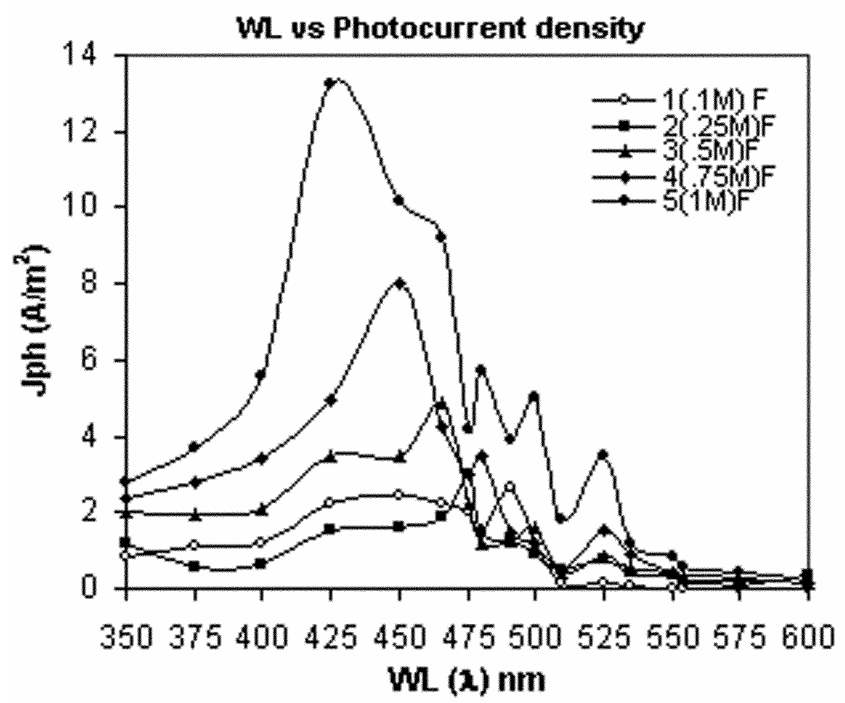

Figure 6. Photocurrent density $\left(J_{\mathrm{ph}}\right)$ vs wavelength $(\lambda)$ plots for CdS films. (Intensity of light normalized at 100 lux.) 
higher molarity. The additional absorption peaks along longer wavelength side is attributed to defects in the films.

\section{Conclusions}

Thin films of CdS prepared by CBD technique in PVA matrix are found to be nanocrystalline. The crystallite sizes measured by XRD studies are found to be within 5.4-10.2 nm, and from SEM, are within 44.4-103.2 $\mathrm{nm}$. The UV absorption studies on films clearly show an increase in band gap which supports the formation of nanocrystallites in these films. A spectral response study was also made to determine the band gap which also reveals similar facts of increase of band gap with the decrease in crystallite size. The overall deposition technique clearly observed that higher molarity facilitates the growth of nanocrystallite in $\mathrm{CdS}$ films in PVA matrix.

\section{Acknowledgement}

One of the authors (RD) thanks UGC for providing a teacher fellowship for the period when the work was being done.

\section{References}

Behera S N, Sahu S N and Nanda K K 2000 Indian J. Phys. A74 81
Breen M L, Woodward J T IV, Schwartz D K and Apblett A W 1998 Chem. Mater. 10710

Britt J and Ferekids C 1993 Appl. Phys. Lett. 622851

Brus L 1986 J. Phys. Chem. 902555

Chu T L, Chu S S, Britt J, Ferekids C, Wang C, Wu C Q and Ullal H S 1992 IEEE Electron. Dev. Lett. EDL-13 303

Dhumure S S and Lokhande C D 1993 Indian J. Pure \& Appl. Phys. 31512

He R et al 2003 Mater. Lett. 571351

Iyechika Y, Wigner G, Jager D, Witt A and Klingshirn C 1988 SPIE Opt. Comput. 88103

Kerk J de and Kelly E F 1964 Appl. Phys. Lett. 52

Kisiel A, Pukowska B and Tomkowicz W 1976 Thin Solid Films 34399

Lee Jae-Hyeong et al 2003 Thin Solid Films 344431

Martin T P et al 1982 Spectrochim. Acta A38 655

Nandakumar P et al 1997 Bull. Mater. Sci. 20579

Periasamy A et al 1997 Proceedings of national conference on spectrophysics (ed.) S Gunasekaran (Chennai: Pachaiyappa's College) p. 95

Sharma T P, Sharma S K and Singh V 1992 CSIO Commun. 1963

Stefko V V 1991 Sov. J. Commun. Technol. Electron. 36

Tang H et al 2005 Mater. Lett. 591024

Tauc J (ed.) 1974 Amorphous and liquid semiconductors (New York: Plenum) p. 159

Tong Hua and Zhu Ying-Jie 2006 Nanotechnology 17845

Williams D H and Fleming I 2004 Spectroscopic methods in organic chemistry (New Delhi: Tata McGraw-Hill) 5th ed., p. 57 